\title{
Role of HLA-G as a Predictive Marker of Low Risk of Chronic Rejection in Lung Transplant Recipients: A Clinical Prospective Study
}

\author{
O. Brugière $\mathrm{e}^{1,2,3,4,5,6, *}, \mathrm{G}$. Thabut $\mathrm{t}^{1,2,3,4}$, \\ I. Krawice-Radanne ${ }^{5,6}$, R. Rizzo ${ }^{7}$, G. Dauriat ${ }^{1,2,3}$, \\ C. Danel ${ }^{1,2,3,8}$, C. Suberbielle ${ }^{9}$, H. Mal ${ }^{1,2,3,4}$, \\ M. Stern ${ }^{10}$, C. Schilte ${ }^{2,4}$, M. Pretolani ${ }^{2,4}$, \\ E. D. Carosella ${ }^{5,6}$ and N. Rouas-Freiss ${ }^{5,6}$ \\ ${ }^{1}$ Service de Pneumologie $B$ et de Transplantation \\ Pulmonaire, Centre Hospitalier Universitaire (CHU) \\ Bichat-Claude Bernard, Paris, France \\ ${ }^{2}$ Faculté de Médecine Denis Diderot, Université Paris 7, \\ Paris, France \\ ${ }^{3}$ Assistance Publique des Hôpitaux de Paris (AP-HP), \\ Paris, France \\ ${ }^{4} \mathrm{DHU}$ Fire, Paris, France \\ ${ }^{5}$ CEA, Institut des Maladies Emergentes et des Therapies \\ Innovantes (IMETI), Service de Recherche en Hemato- \\ Immunologie (SRHI), Hopital Saint-Louis, Paris, France \\ ${ }^{6}$ Sorbonne Paris Cité, University Paris Diderot, IUH, \\ Hopital Saint-Louis, Paris, France \\ ${ }^{7}$ Department of Medical Sciences, Section of \\ Microbiology and Medical Genetics, University of Ferrara, \\ Ferrara, Italy \\ ${ }^{8}$ Service d'Anatomie et Cytologie Pathologiques, CHU \\ Bichat-Claude Bernard, Paris, France \\ ${ }^{9} \mathrm{CHU}$ Saint-Louis, Paris, France \\ ${ }^{10}$ Service de Pneumologie, Hôpital Foch, Paris, France \\ * Corresponding author: Olivier Brugière, \\ olivier.brugière@bch.aphp.fr
}

Human leukocyte antigen G (HLA-G) expression is thought to be associated with a tolerance state following solid organ transplantation. In a lung transplant (LTX) recipient cohort, we assessed (1) the role of HLA-G expression as a predictor of graft acceptance, and (2) the relationship between (i) graft and peripheral HLA-G expression, (ii) HLA-G expression and humoral immunity and (iii) HLA-G expression and lung microenvironment. We prospectively enrolled $63 \mathrm{LTx}$ recipients (median follow-up 3.26 years [min: 0.44-max: 5.03]). At 3 and 12 months post-LTx, we analyzed graft HLA-G expression by immunohistochemistry, plasma soluble HLA-G (sHLA-G) level by enzyme-linked immunosorbent assay, bronchoalveolar lavage fluid (BALF) levels of cytokines involved in chronic lung allograft dysfunction (CLAD) and anti-HLA antibodies (Abs) in serum. In a time-dependent Cox model, lung HLA-G expression had a protective effect on CLAD occurrence (hazard ratio: 0.13 [0.03-0.58]; $p=0.008$ ). The same results were found when computing 3-month and 1-year conditional freedom from CLAD ( $p=0.03$ and 0.04 , respectively [log-rank test]). Presence of anti-HLA Abs was inversely associated with graft HLA-G expression $(p=0.02)$. Increased BALF level of transforming growth factor- $\beta$ was associated with high plasma sHLA-G level $(p=0.02)$. In conclusion, early graft HLA-G expression in LTx recipients with a stable condition was associated with graft acceptance in the long term.

Abbreviations: Ab, antibody; APC, antigen-presenting cell; AR, acute rejection; BALF, bronchoalveolar lavage fluid; BECs, bronchial epithelial cells; BOS, bronchiolitis obliterans syndrome; $\mathrm{CO}$, predose concentration; $\mathrm{C2}$, 2-h postdosage concentration; CLAD, chronic lung allograft dysfunction; CMV, cytomegalovirus; ELISA, enzymelinked immunosorbent assay; $F E V_{1} / F V C$ ratio, forced expiratory volume in 1 s/forced vital capacity; GM-CSF, granulocyte-macrophage colony-stimulating factor; HLA-G, human leukocyte antigen G; HLA-G+, HLA-G positive; HLA-G-, HLA-G negative; IFN- $\gamma$, interferongamma; IHC, immunohistochemical; IP-10, interferon gamma induced protein 10; ISHLT, International Society for Heart and Lung Transplantation; i.v., intravenous(ly); LTx, lung transplant(ion); MCP1, monocyte chemoattractant protein-1; MIG, monokine-induced by gamma interferon; NK, natural killer; NS, not significant; pg, picogram(s); RAS, restrictive allograft syndrome; SAFB, single-antigen flow-beads; $S D$, standard deviation; sHLA-G, soluble HLA-G; TBBx, transbronchial biopsies; TGF- $\beta$, transforming growth factor- $\beta$; TLC, total lung capacity; TNF- $\alpha$, tumor necrosis factor- $\alpha$

Received 13 December 2013, revised 04 August 2014 and accepted for publication 07 August 2014

\section{Introduction}

Lung transplantation ( $\mathrm{LTX}$ ) is now a widely accepted procedure for end-stage pulmonary diseases. Nevertheless, long-term survival still remains limited because of chronic lung allograft dysfunction (CLAD), thought to represent a form of chronic rejection. CLAD is the main complication after LTX and remains the most common cause of graft failure and death (1).

Currently, the available immunosuppressive therapies cannot prevent the occurrence of CLAD in many patients. Conversely, some LTX recipients, under standard 


\section{Brugière et al}

immunosuppression therapy, remain free of CLAD for a long time, as reported for other solid organ transplantations (2). Better understanding of a tolerogenic state in some patients and identifying markers of long-term functional stability are needed. During the past decade, attention has been paid to the human leukocyte antigen $G$ (HLA-G) molecule, a nonclassical HLA class I molecule, reported as a critical immunosuppressive molecule (3). HLA-G can be expressed as both membrane-bound proteins (HLA-G1, HLA-G2, HLA-G3 and HLA-G4) and soluble isoforms (HLA-G5, HLA-G6 and HLAG7) (3). HLA$\mathrm{G}$ has a low polymorphism and its expression is highly tissue-restricted: Besides being expressed in fetal tissues, such as trophoblast cells, HLA-G constitutive expression is found only in adult thymic medulla, cornea, pancreatic islets and erythroid and endothelial cell precursors (4). Given the HLA-G expression patterns, its prime physiologic relevance is likely to be at the fetal-maternal interface (5), as a key contributor to the tolerance of the fetus by the immune system of the mother. Nevertheless, HLA-G expression can also be induced in various pathologic conditions, including solid organ Tx, malignant transformations, viral infections and inflammatory and autoimmune diseases (3). In organ Tx, HLA-G has been detected in both graft biopsies and sera in heart, kidney and/or liver Tx, and its expression was found associated with allograft acceptance, displayed as fewer episodes of acute rejection (AR) and no chronic rejection (6-13). In this regard, HLA-G possesses widely described immune tolerogenic properties by modulating the functions of several immune effectors such as natural killer (NK) cells, T cells and antigen-presenting cells through direct binding to the inhibitory receptors immunoglobulin (lg)-like transcript 2 (ILT-2; LILRB1/CD85j), ILT-4 (LILRB2/ CD85d) and killer cell Ig-like receptor 2DL4 (KIR2DL4; CD158d) $(3,14,15)$. In vitro data indicate that HLA-G inhibits both NK cell and CD8+ T cell mediated cytolysis (16), suppresses CD4+ $\mathrm{T}$ cell alloproliferative responses (17) and induces apoptosis of CD8+ T cells (18).

We previously reported increased expression of HLA-G in the bronchial epithelium of some LTx recipients, which was associated with a stable condition at the date of biopsies (19). In the present study, we aimed to ascertain the prognostic value of HLA-G expression in the graft and/or plasma to identify LTx recipients with low immunologic risk of subsequent chronic rejection. This prospective clinical study aimed to determine whether early expression of HLA$G$ in LTx recipients could be a predictor of the status of rejector or nonrejector status at long-term follow-up (as determined by the occurrence of CLAD). Furthermore, we also examined (i) the correlation between in situ graft expression of HLA-G and plasma level of soluble HLA-G (sHLA-G), (ii) the relationship between graft HLA-G expression and humoral immunity, reflected by the detection of anti-HLA antibodies (Abs) and (iii) the link between graft or plasma HLA-G expression and the lung microenvironment, that is, the cytokine profile in bronchoalveolar lavage fluid (BALF).

\section{Patients and Methods}

\section{Patients}

We enrolled 63 patients in this prospective study. All patients gave their informed consent for participation in the study. Patients underwent LTX in Bichat or Foch hospitals (Paris) from November 2009 to December 2011.

At 3-6 months (visit 1, V1) and 12 months (visit 2, V2) post-LTx, patients considered in clinical stable condition underwent planned systematic transbronchial biopsies (TBBX), BALF sampling during fiber-optic endoscopy and $5 \mathrm{~mL}$ plasma sampling. A stable condition was defined as the lack of new respiratory symptoms or new radiographic findings. BALF was systematically used to determine the presence of bacteria, viruses and fungi (20) in the two enrolling centers. Bacterial colonization with previously defined criteria (20), was not considered a criterion of exclusion. Multiplex polymerase chain reaction assay was used to detect respiratory viruses in BALF. All TBBx specimens were systematically assessed for rejection, and an episode of AR was diagnosed histologically and graded by International Society for Heart and Lung Transplantation (ISHLT) criteria (21). In parallel, graft HLA-G expression in TBBx specimens was analyzed by immunochemistry, and plasma levels of sHLA-G (stored at $-80^{\circ} \mathrm{C}$ ) were examined by enzyme-linked immunosorbent assay (ELISA). TBBx specimens were not analyzed in cases of viral infection with cytomegalovirus (CMV), herpes or influenza A viruses detected in BALF because these viruses are specifically known to up-regulate HLA-G expression in bronchial epithelium (19,22-24). An AR score was defined by the number of biopsyproven cellular AR episodes during the first 12 months after LTx (25). Patients with CLAD were classified by two clinical phenotypes: bronchiolitis obliterans syndrome (BOS) defined by the classical definition of the ISHLT (26) or restrictive allograft syndrome (RAS) (27). RAS was defined as a decline in total lung capacity (TLC) of at least $10 \%$ from baseline, but in case not enough TLC measurements were available, a forced expiratory volume in $1 \mathrm{~s} /$ forced vital capacity $\left(F E V_{1} / F V C\right)$ index that remained normal or increased above normal with an FVC decline of at least $20 \%$ from baseline was also considered restrictive, as used previously (27) whereas a FEV $1 / F V C$ index of less than 0.7 was considered obstructive. At each visit, patients followed-up at Bichat Hospital were screened for anti-HLA Abs by Luminex assay (One Lambda, Canoga Park, CA). Abs detected against HLA Class I and Class II antigen were identified by single-antigen flow-beads (SAFB) Luminex assay (One Lambda, Canoga Park, CA). All patients were followed at least every 3 months, and for those with pulmonary complications, additional fiber-optic bronchoscopy for BALF and TBBx sampling was performed to determine clinical, physiological or radiographic changes.

\section{Immunosuppression}

All LTx recipients received maintenance immunosuppressive therapy with cyclosporine ( $n=49$; Bichat Hospital) or tacrolimus ( $n=14$; Foch Hospital), mycophenolate mofetil ( $2 \mathrm{~g} /$ day) and prednisolone (500-mg intravenous [i.v.] methylprednisolone before operation and before reperfusion of the graft; $0.5 \mathrm{mg} / \mathrm{kg} /$ day on the following days and thereafter replaced by oral prednisone, which was progressively tapered to $0.1 \mathrm{mg} / \mathrm{kg} /$ day after 12 weeks). In Foch Hospital ( $n=14$ patients), rabbit antithymocyte globulin induction therapy was given during the postoperative period $(1.5 \mathrm{mg} / \mathrm{kg} / \mathrm{day}$ for 3 days), except for CMV-infection-negative recipients with CMVinfection-positive donors. Cyclosporine monitoring involved the 2-h postdosage concentration (C2) with target C2 levels of 1200 and $800 \mathrm{~kg} / \mathrm{L}$ at 1 week and 3 months, respectively. Tacrolimus monitoring involved the predose concentration (CO), with target predose concentration levels of 8 $13 \mathrm{ng} / \mathrm{mL}$. AR episodes $\geq \mathrm{A} 1$ (20) were treated with i.v. methylprednisolone $(15 \mathrm{mg} / \mathrm{kg} / \mathrm{day})$ for 3 days, then an oral taper of prednisolone. Steroidresistant $A R$ was treated with rabbit antithymocyte globulin therapy $(2.5 \mathrm{mg} /$ $\mathrm{kg} /$ day for 5 days) in case of failure of a 3-day course of i.v. methylprednisolone $(15 \mathrm{mg} / \mathrm{kg} /$ day). 
This study was approved by an institution ethics committee, and conducted in accordance with good clinical practices and the recommendations concerning human research contained in the Declaration of Helsinki. All patients gave their informed consent to be included in the study.

\section{Histology and immunohistochemistry}

Lung TBBx specimens were fixed in 10\% buffered formalin and embedded in paraffin. Sections $4 \mu \mathrm{m}$ thick underwent hematoxylin and eosin, Masson's trichrome or periodic acid-Schiff staining. All TBBx specimens were examined by a single LTx pathologist who was blinded to patients' clinical status. In parallel with routine histological examination, all paraffinembedded TBBX specimens were assessed for HLA-G expression by immunohistochemical (IHC) analysis, as described (19), by use of the MEMG/02 mAb at 1:50 dilution (Exbio, Praha, Czech Republic), which recognizes all HLA-G isoforms. An isotype-matched Ab was used as a control for nonspecific staining. Trophoblast tissue sections and lung specimen sections considered normal were used as positive and negative controls, respectively, for HLA-G staining. IHC analysis involved the Ultratech HRP Streptavidin-Biotin Universal Detection System (Immunotech-Coulter, Roissy, France). Briefly, deparaffinized tissue sections underwent epitope retrieval by high temperature in $10 \mathrm{mmol} / \mathrm{L}$ sodium citrate buffer $(\mathrm{pH} \mathrm{6.0)}$ in a commercial microwave oven to optimize immunoreactivity. The slides were rehydrated for $5 \mathrm{~min}$ in phosphate-buffered saline containing $0.1 \%$ saponin and $10 \mathrm{mmol} / \mathrm{L}$ HEPES buffer. Endogenous peroxidase activity was quenched by treating the sections for $5 \mathrm{~min}$ at room temperature with $3 \%$ hydrogen peroxide in water. Nonspecific binding was prevented by use of $20 \%$ human serum for 20 min before staining with the mAb for $30 \mathrm{~min}$ at room temperature. Immunostaining was evaluated by use of the EnVision System Peroxidase (AEC; Dako, Les Ulis, France). All tissues were counterstained with hematoxylin. A specimen was considered positively labelled for bronchial epithelial cells (BECs) if at least $20 \%$ of BECs in the tissue section contained visually detectable red chromogen in the membrane and/or cytoplasm (15). Both bronchiolar and bronchial epithelia were considered for the analysis. We graded the intensity of staining from to $+++(-,+,++,+++)$ as performed in previous work (15) and considered a positive staining for all cases with grading from + to +++ .

\section{Classification of patients by HLA-G expression in the graft lung} Patients were classified as positive for bronchial epithelium expression of HLA-G (lung HLA-G+) if at least one of TBBx at V1 or V2 was considered HLA-G+ with MEM-G/2 mAb staining. The remaining patients were considered negative for lung HLA-G expression (lung HLA-G-). Two patients were not classified because of viral infection at the time of available TBBx. These two patients were free of CLAD at the last-follow-up.

\section{Plasma sHLA-G level measured by ELISA}

Plasma sHLA-G level was measured by ELISA as reported (28) with the MAb G233 (Exbio, Praha, Czech Republic), which recognizes the HLA-G molecule in a $\beta_{2}$-microglobulin-associated form. The intra- and inter-assay coefficients of variation were $1.4 \%$ and $4.0 \%$, respectively. The limit of sensitivity was $1.0 \mathrm{ng} / \mathrm{mL}$. sHLA-G was measured in LTX recipients at V1 and V2 and in 63 normal-health volunteers matched by sex and age.

\section{Cytokine and chemokine analysis in BALF}

We analyzed levels of cytokines and chemokines known to be elevated in the BALF of LTX recipients with BOS (29): IL-6, IL-8 and IL-17; monocyte chemoattractant protein-1 (MCP1; chemokine ligand [CCL] 2); monokineinduced by gamma interferon (MIG; CXCL9); interferon gamma induced protein 10 (IP-10; CXCL10); and RANTES. We also analyzed BALF levels of cytokines previously shown to up-regulate HLA-G, including IL-10, IL-13, granulocytemacrophage colony-stimulating factor (GM-CSF), tumor necrosis factor- $\alpha$
(TNF- $\alpha$ ), interferon gamma (IFN- $\gamma$ ) and TGF- $\beta$ (3). TGF- $\beta$ and CXCL9 were quantified by ELISA (R\&D duoset; R\&D System, Abingdon, UK), and other analytes were quantified by Luminex (Millipore HCYTOMAG-60K and HCYP2MAG-62K; Merck Millipore, Suresnes, France) with a magpix device. Lower limits of detection, determined with the exponent software, were as follows: IL-8 [0.6 picogram $(\mathrm{pg}) / \mathrm{mL}], \mathrm{CCL} 2(10.8 \mathrm{pg} / \mathrm{mL}), \mathrm{IL}-6(0.2 \mathrm{pg} / \mathrm{mL})$, CXCL9 (7 pg/mL), IP-10 (3 pg/mL), GM-CSF(0.1 pg/mL), TNF- $\alpha(1.3 \mathrm{pg} / \mathrm{mL}), \mathrm{IFN}-$ $\gamma(0.1 \mathrm{pg} / \mathrm{mL})$, TGF- $\beta(5 \mathrm{pg} / \mathrm{mL})$ and IL-13 $(0.2 \mathrm{pg} / \mathrm{mL})$. Profiles of cytokines and chemokines were compared between lung HLA-G+ and lung HLA-Gpatients, and between patients with high and those with low sHLA-G trough level in plasma.

\section{Statistical analysis}

Continuous variables are described by their mean and $S D$, or median and ranges, and compared by use of Student's t-test or Mann-Whitney U-test. Categorical variables are described by frequencies and percentages and compared by chi-square test. Correlations between continuous variables were assessed by Spearman's rank correlation coefficient. Some analyses involved measurements at two time points for the same patient. In such cases, random-effect models (random intercept) were fitted to account for the within-patient correlation. Time to CLAD onset and time to death or retransplantation $(n=1)$ were estimated by the Kaplan-Meier estimator and compared by log-rank test. Univariate and multivariable Cox models were built to assess the relationship between early HLA-G staining and time to CLAD onset while adjusting for potential confounding factors. In these Cox models, HLA-G staining was included as a time-dependent covariate. Factors associated with CLAD occurrence by univariate analyses (at a significance of $p<0.2$ ) were selected for multivariate analyses. We computed 3-month and 1-year conditional survivals including only patients who were alive and free of CLAD at these time points $(n=61$ and $n=54$, respectively). Because of the limited number of events (death or retransplantation), no multivariable analysis was carried out for survival. The last follow-up date for all survival models was March 2014. For all analyses, $p<0.05$ was considered statistically significant. Analyses involved the use of Stata v12 for Macintosh (StataCorp LP, College Station, TX).

\section{Results}

\section{Characteristics of patients}

Among the 63 patients (mean [SD] age, 49 [12] years; 19 female), 31 (49\%) underwent bilateral LTx and 32 (51\%) single LTx (Table 1). The initial diagnosis was emphysema $(n=19)$, pulmonary fibrosis $(n=25)$, cystic fibrosis $(n=11)$ and other $(n=8)$. Mean ischemic time was 358 (SD: 163) min. Median follow-up was 3.26 years (min: 0.44-max: 5.03). At last follow-up, 19 patients (30\%) had CLAD with severe graft failure due to CLAD in 13 patients (20\%) (eight patients had died from CLAD, one had undergone re-TX, and four were still alive, with Class IV New York Heart Association-defined dyspnea and oxygen dependency). Among patients with CLAD, 15 had a BOS phenotype and 4 a RAS phenotype. Baseline characteristics of the entire cohort are detailed in Table 1.

\section{Graft acceptance by HLA-G immunostaining in lung graft}

Classification of patients by in situ HLA-G immunostaining: We had 108 available TBBx specimens for 63 patients for $H L A-G$ immunostaining, which allowed for classifying lung HLA-G+ and $H L A-G$ - patients (Figure 1). A 


\section{Brugière et al}

Table 1: Baseline characteristics of 63 patients who underwent LTx by presence or not of HLA-G expression in the grafted lung

\begin{tabular}{|c|c|c|c|c|}
\hline Variable & Entire cohort $(n=63)$ & Lung HLA-G $+{ }^{1}(n=31)$ & Lung HLA-G- ${ }^{1}(n=30)$ & p-Value \\
\hline Age, mean (SD) & $49.6(12.5)$ & $47.8(12.1)$ & $51.4(12.8)$ & 0.25 \\
\hline Female, n (\%) & $18(29.5)$ & $9(29.0)$ & $9(30.0)$ & 0.93 \\
\hline Diagnosis, n (\%) & - & - & - & 0.39 \\
\hline COPD or A1-AT & $19(30)$ & $10(33)$ & $8(26)$ & - \\
\hline Cystic fibrosis & $13(21)$ & $6(19)$ & $6(19)$ & - \\
\hline Fibrosis & $25(40)$ & $10(32)$ & $15(50)$ & - \\
\hline Other & $6(9)$ & $5(16)$ & $1(3)$ & - \\
\hline Bilateral LTx, n (\%) & $29(46)$ & $18(58)$ & $11(37)$ & 0.09 \\
\hline Ischemic time: $\min (\mathrm{SD})$ & 351 (159) & 364 (126) & 338 (189) & 0.54 \\
\hline CMV status & - & - & - & 0.65 \\
\hline $\mathrm{D}+$ or $-/ \mathrm{R}+$ & $36(61)$ & $17(59)$ & 19 (63) & - \\
\hline $\mathrm{D}-/ \mathrm{R}-$ & $20(34)$ & $10(34)$ & $10(33)$ & - \\
\hline $\mathrm{D}+/ \mathrm{R}-$ & $3(5)$ & $2(7)$ & $1(3)$ & - \\
\hline \multicolumn{5}{|l|}{ IS therapy } \\
\hline \multicolumn{5}{|l|}{ Tacrolimus, n (\%) } \\
\hline $\mathrm{V} 1: \mathrm{C} 0, \mathrm{ng} / \mathrm{mL}$ & $8.9(3.1)$ & $9.0(3.1)$ & $8.8(3.0)$ & 0.86 \\
\hline V2: $\mathrm{CO} \mathrm{ng} / \mathrm{mL}$ & $8.9(3.1)$ & $8.8(2.4)$ & $9.1(3.9)$ & 0.78 \\
\hline \multicolumn{5}{|l|}{ Cyclosporine, n (\%) } \\
\hline $\mathrm{V} 1: \mathrm{C} 2, \mathrm{ng} / \mathrm{mL}$ & $1119(378)$ & $1269(378)$ & $1044(290)$ & 0.27 \\
\hline $\mathrm{V} 2: \mathrm{C} 2, \mathrm{ng} / \mathrm{mL}$ & $944(196)$ & $1054(164)$ & $871(209)$ & 0.38 \\
\hline \multicolumn{5}{|l|}{ Steroids, mg, mean (SD) } \\
\hline $\mathrm{V} 1: \mathrm{mg}$, mean (SD) & $22(8)$ & $22(9)$ & $23(6)$ & 0.83 \\
\hline V2: mg, mean (SD) & $8(2)$ & $8(2)$ & $8(3)$ & 0.82 \\
\hline \multicolumn{5}{|l|}{ Inf. colonization, n (\%) } \\
\hline Bacterial V1 & $16(28)$ & $8(14)$ & $8(14)$ & 0.46 \\
\hline Fungal $\mathrm{V}_{1}$ & $5(9)$ & $4(7)$ & $1(2)$ & - \\
\hline Bacterial + fungal V1 & $3(5)$ & $2(3)$ & $1(2)$ & - \\
\hline Bacterial V2 & $11(26)$ & $2(5)$ & $9(21)$ & 0.056 \\
\hline Fungal V2 & $3(7)$ & $3(7)$ & 0 & - \\
\hline Bacterial + fungal V2 & $2(5)$ & $1(2)$ & $1(2)$ & - \\
\hline Follow-up, median, (range), year & - & $3.26(0.44-5.03)$ & $3.49(0.46-4.83)$ & 0.5 \\
\hline
\end{tabular}

A1-AT, antitrypsin deficiency emphysema; BOS, bronchiolitis obliterans; COPD, chronic obstructive pulmonary disease; LTX, lung transplantation; CMV, cytomegalovirus; CMV status: $\mathrm{D}+$ or $-/ \mathrm{R}+$, donor-positive or -negative/recipient-positive; $\mathrm{D}-/ \mathrm{R}-$, donor-negative/ recipient-negative; $\mathrm{D}+/ \mathrm{R}-$, donor-positive/recipient-negative; IS, immunosuppression.

Cyclosporine monitoring involved 2-h postdose concentration (C2) and tacrolimus monitoring involved the predose concentration (C0). Inf. Colonization: infectious colonization, with previously defined criteria of infectious colonization (20). Patients were classified as lung HLA-G positive (HLA-G+) if at least one of transbronchial biopsies (TBBx) at visit 1 (V1 at 3 months) or visit 2 (V2 at 12 months) was considered HLA$\mathrm{G}$ positive, with bronchial epithelium stained with MEM-G/2 mAb. The remaining patients were considered lung HLA-G negative (HLA-G-). ${ }^{1}$ Two patients could not be classified because of Bx grading according to the ISHLT classification (21) or missed biopsy schedule at both V1 and $\mathrm{V} 2$.

panel of positive and negative staining of the bronchial epithelium from different patients is shown in Figure 2, as previously described (19).

In total, 99 TBBx were included in the final analysis to classify 31 patients as lung HLA-G+ and 30 as HLA-G(Figure 1). Two patients could not be classified (Figure 1, Table 2). Classification according to the ISHLT (21) showed 83 TBBx graded A0B0 ( $n=34 \mathrm{HLA}-\mathrm{G}+$ and $n=49 \mathrm{HLA}-$ G-), 9 TBBx graded A1/2B0 ( $n=3 \mathrm{HLA}-\mathrm{G}+; n=6 \mathrm{HLA}-\mathrm{G}-$ ) and $n=7$ TBB $\times$ B $1 / 2$ regardless of concurrent grade $A$ score ( $n=5 \mathrm{HLA}-\mathrm{G}+$ and $\mathrm{n}=2 \mathrm{HLA}-\mathrm{G}-$ ). We found no significant association of pathology grade and $\mathrm{HLA}-\mathrm{G}$ staining $(p=0.24)$. HLA-G expression was not analyzed in cases with viral infection known to induce HLA-G expression in the bronchial epithelium at sampling (5 lung HLA-G+ and 4 lung HLA-G - patients had CMV $[n=4]$, herpes $[n=3$ ] or influenzae $A[n=2]$ infection diagnosed in BALF at $V 1$ or V2). Two of the 63 patients remained unclassified according to HLA-G staining of TBBx, because of Bx grading (21) or missed biopsy schedule at $\mathrm{V} 1$ and $\mathrm{V} 2$.

Graft survival, freedom from CLAD, and AR score: The median (range) duration of follow-up did not differ between $\mathrm{HLA}-\mathrm{G}+$ and $\mathrm{HLA}-\mathrm{G}-$ patients (3.26 years [0.44-5.03] and 3.49 years [0.46-4.84], respectively, $p=0.5)$, nor did mean (SD) AR scores differ according to HLA-G staining (1.20 [1.13] vs. 1.48 [1.58]; $p=0.45)$.

In the total cohort, freedom from CLAD was 88.7\% $(80.6-$ $97.6 \%), 65.4 \%$ (53.7-79.8\%) and 63.1\% (51.1-77.9\%) at 1,3 and 5 years, respectively. Figures 3 and 4 show the 


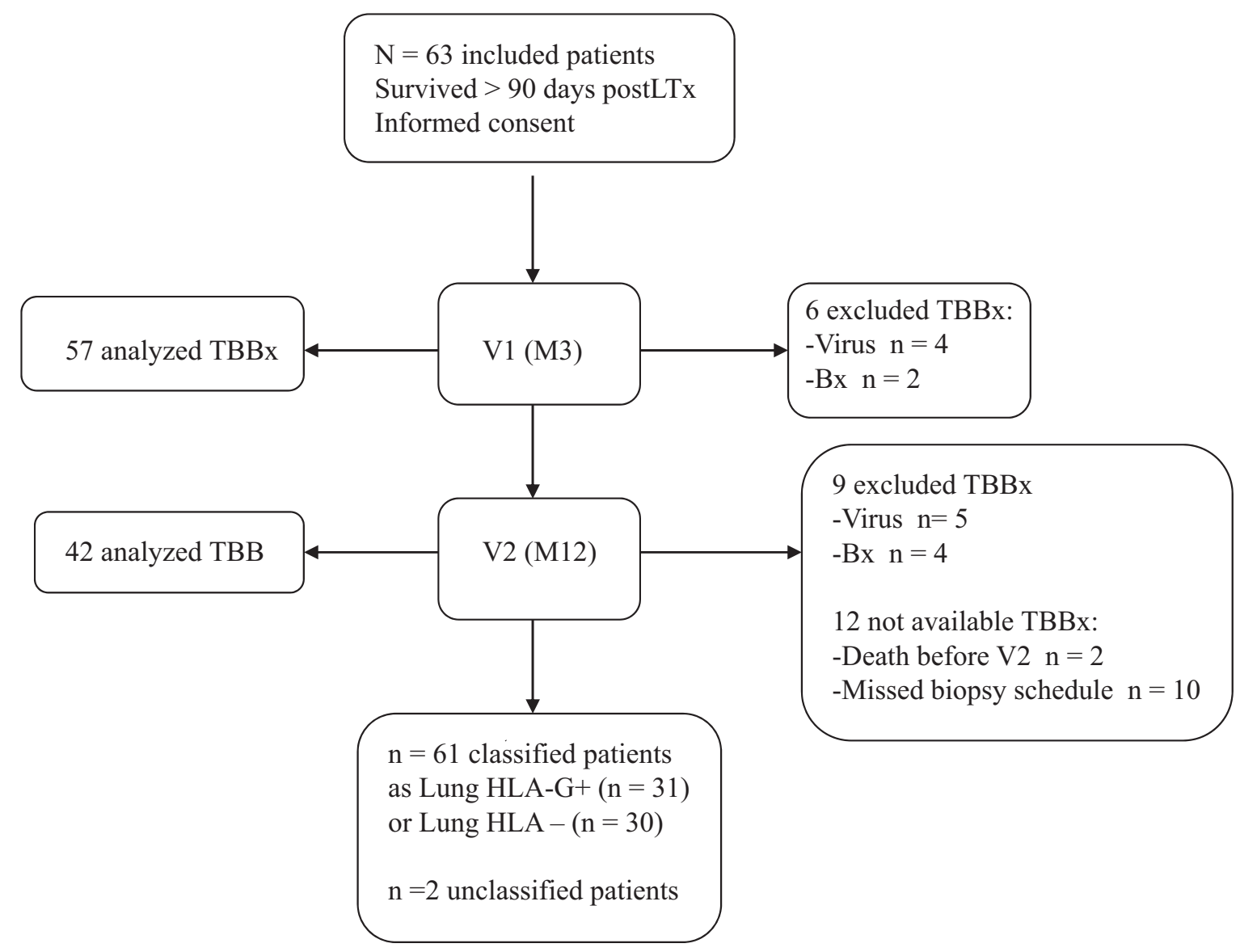

Figure 1: Study flow chart.

proportion of patients free of CLAD conditional on being alive and free of CLAD at 3 and 12 months after LTX, respectively.

The proportion of patients free of CLAD computed from 3 months post-LTx was 90.9\% [79.7-100] and 81.6\% [66.8-99.7] at 1 and 3 years, respectively, among lung HLA$\mathrm{G}+$ patients, as compared with 75.0\% [60.6-92.9\%] and $52.1 \%$ [36.2-75.1\%], respectively, among lung HLA-Gpatients (log-rank, $\mathrm{p}=0.03$ ) (Figure 3 ). The proportion of patients free of CLAD computed from 12 months post-LTX was 86.4\% [73.2-100] and 81.6\% [66.8-99.7] at 1 and 3 years, respectively, among lung HLA-G+ patients, as compared with 78.3\% [63.1-97.1\%] and 63.5\% [46.0$87.5 \%$ ], respectively, among lung HLA-G - patients (logrank, $p=0.04$ ) (Figure 4).

In a univariate Cox model incorporating HLA-G staining measured at 3 and 12 months post-LTx as a timedependent covariate and stratified on center, positive graft HLA-G staining was associated with a reduced risk of CLAD development (hazard ratio [HR]: 0.17 [0.05-0.60]; $p=0.001$ ) (Table 3 ). When other factors associated with
CLAD occurrence in univariate analyses at a significance level of 0.2 were included in a multivariable Cox model, the sole independent predictive factor associated with a reduced risk of CLAD development was graft HLA-G staining (HR: 0.13 [0.03-0.58]; $p=0.008$ ) (Table 3).

Finally, in a Cox model performed for only the single center where anti-HLA Abs were screened $(n=49), \quad H L A-G$ expression still had a protective effect on CLAD occurrence, whether the model included anti-HLA Abs (HR: 0.132 [0.0285-0.6107]; $p<0.01$ ) or not (HR: 0.126 [0.028$0.576] ; p<0.01)$.

Furthermore, when considering CLAD status at last followup, $62 \%$ of patients free of CLAD $(n=26 / 42)$ versus only $26 \%$ with CLAD $(n=5 / 19)$ were graft HLA-G+ $(p<0.05)$. Survival was $96.7 \%$ (92.4-100\%), 88.2\% (80.4-96.8\%) and $68.9 \%(53.8-88.1 \%)$ at 1,3 and 5 years, respectively. Time to death or retransplantation estimated by the KaplanMeier estimator and compared by log-rank test was similar for lung HLA-G+ and HLA-G- patients $(p=0.76)$. Because of the limited number of events, we did not perform multivariable analysis for survival. 


\section{Brugière et al}

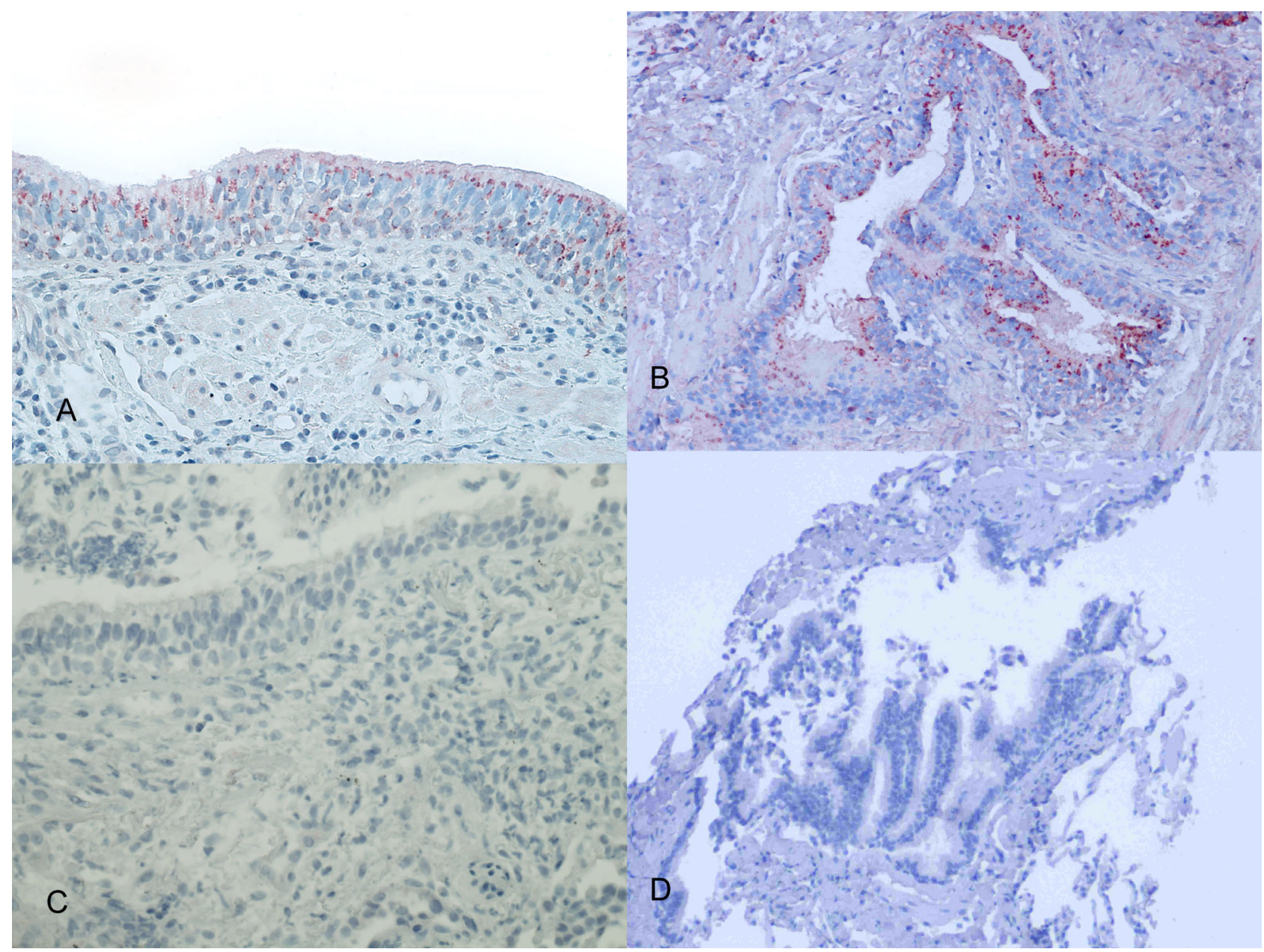

Figure 2: Examples of human leukocyte antigen G (HLA-G) staining in lung tissue specimens from lung transplant recipients. The MEM-G/2 mAb was used to detect HLA-G in bronchial epithelial cells of lung tissues obtained from transbronchial biopsies. (A, B) Staining of HLA-G in bronchial epithelial cells from two different patients. The cytoplasm of the bronchial or bronchiolar epithelial cells is strongly labelled by anti-HLA-G antibody. (C, D) Negative staining in bronchial epithelium from two different patients. Original magnification $\times 200$.

Lung HLA-G+ and HLA-G- patients did not differ in any factors that might influence HLA-G expression in the graft, including recipient and donor characteristics lage and sex of recipients, initial disease, LTx procedure, CMV serologic status of donor and recipient), dosage of prednisone, trough levels of tacrolimus or cyclosporine, bronchial infection and duration of follow-up (Table 1).

\section{Association of plasma sHLA-G level and graft acceptance}

First, we compared mean trough level of sHLA-G in plasma from the 63 LTx recipients to that of 63 normal-health volunteers (males or nonparous female) matched by sex and age. Median (range) plasma level of sHLA-G was significantly higher for LTx recipients than healthy volunteers (5.6 [0-72.2] vs. 0 [0-48.9]; $p<0.05)$. In the LTx cohort, median plasma level of sHLA-G in plasma at V1 did not differ between patients with and without CLAD at lastfollow-up (9.2 [range: 0-67.0] vs. 0 [0-36.6] ng/mL at V1, $p=0.24)$, but was significantly higher at $V 2$ for patients with than without subsequent CLAD at last follow-up (14.5 [072.2] vs. 4.6 [0-36.1]; $p=0.04)$.

\section{Relationship between graft and sHLA-G expression}

Mean (SD) plasma level of SHLA-G at V1 and V2 were similar between lung HLA-G+ and HLA-G- patients $(9.7$ [2.3] vs. 13.4 [3.0] $\mathrm{ng} / \mathrm{mL}$ at $\mathrm{V} 1 ; p=0.34$, and 11.4 [3.2] vs. 10.4 [2.2] $\mathrm{ng} / \mathrm{mL}$ at $\mathrm{V} 2 ; \mathrm{p}=0.79$ ), showing no association of graft HLA-G expression and peripheral sHLA-G secretion.

\section{Relationship between graft HLA-G expression and anti-HLA abs}

Anti-HLA Abs were screened in the 49 patients followed at Bichat hospital. Preformed anti-HLA Abs, de novo anti-HLA Abs at 3 months (V1) and de novo anti-HLA at 12 months (V2) were detected in 25/44 (57\%), 30/49 (61\%) and 21/32 (67\%) patients with available sera, respectively. In addition, 
HLA-G Is Associated With Lung Graft Acceptance

Table 2: Classification of patients ${ }^{1}$ by repeated HLA-G expression testing in graft tissue

\begin{tabular}{|c|c|c|c|c|c|c|}
\hline & $\begin{array}{l}\text { Lung HLA-G + } \\
\text { patients } \\
(n=31)\end{array}$ & & & $\begin{array}{l}\text { Lung HLA-G - } \\
\text { patients } \\
(\mathrm{n}=30)^{*}\end{array}$ & & \\
\hline$n$ & $\begin{array}{l}\text { V1 } \\
\text { (3 mo post-TX) }\end{array}$ & $\begin{array}{l}\text { V2 } \\
(12 \text { mo post-Tx })\end{array}$ & $\mathrm{n}$ & $\begin{array}{l}\text { V1 } \\
\text { (3 mo post-TX) }\end{array}$ & $\begin{array}{l}\text { V2 } \\
(12 \text { mo post-Tx })\end{array}$ & \\
\hline 1 & ++ & ++ & 32 & & & \\
\hline 2 & ++ & ++ & 33 & & & \\
\hline 3 & ++ & ++ & 34 & & & \\
\hline 4 & +++ & +++ & 35 & & NA & \\
\hline 5 & ++ & + & 36 & & & \\
\hline 6 & & + & 37 & virus & & \\
\hline 7 & + & ++ & 38 & & & \\
\hline 8 & + & & 39 & & & \\
\hline 9 & + & virus & 40 & & & \\
\hline 10 & + & + & 41 & & & \\
\hline 11 & ++ & ++ & 42 & & & \\
\hline 12 & + & +++ & 43 & & virus & \\
\hline 13 & +++ & NA & 44 & & & \\
\hline 14 & ++ & ++ & 45 & & & \\
\hline 15 & +++ & NA & 46 & & virus & \\
\hline 16 & ++ & NA & 47 & & & \\
\hline 17 & +++ & ++ & 48 & & NA & \\
\hline 18 & virus & +++ & 49 & & & \\
\hline 19 & + & NA & 50 & & & \\
\hline 20 & + & NA & 51 & virus & & \\
\hline 21 & ++ & NA & 52 & & NA & \\
\hline 22 & ++ & NA & 53 & & NA & \\
\hline 23 & ++ & + & 54 & & & \\
\hline 24 & & + & 55 & & NA & \\
\hline 25 & & ++ & 56 & & & \\
\hline 26 & virus & +++ & 57 & & & \\
\hline 27 & ++ & + & 58 & & & \\
\hline 28 & + & NA & 59 & & & \\
\hline 29 & + & virus & 60 & & & \\
\hline 30 & ++ & virus & 61 & & NA & \\
\hline 31 & & ++ & & & & \\
\hline
\end{tabular}

Patients were classified as lung HLA-G+ (positive) patients if TBBx at visit 1 at 3 months (V1) or at visit 2 at 12 months (V2) were considered HLA-G positive, with bronchial epithelium stained with MEM-G/2 mAb. The remaining patients were considered lung HLA-G negative. Positive HLA-G staining in bronchial epithelium is indicated in gray, and negative HLA-G staining by no color. Intensity of staining was graded from + to +++ . NA: No available specimen. Virus: specimens not analyzed because of concommittent documented viral infection at the time of sampling. HLA-G, human leukocyte antigen G; TBBx, transbronchial biopsies.

*Two patients were not classified because of Bx grading or missed biopsy schedule both at V1 and V2.

n, numbers of patients. Numbers of patients with chronic graft dysfunction at last follow-up are indicated in yellow.

donor-specific Abs were detected in $7 / 44(16 \%)$ patients before LTX, $9 / 49(18 \%)$ patients at 3 months and $8 / 32$ (25\%) patients at 12 months. Among these 49 patients, 72 sera sampled at $\mathrm{V} 1$ and/or $\mathrm{V} 2$ at the same time of TBBx were tested with SAFB assay. SAFB-Luminex-detected anti-HLA Abs were more frequently found in lung HLA-G - than HLA$\mathrm{G}+$ patients $(31 / 38[81.5 \%]$ vs. 18/34 [52.9\%], [odds ratio $=0.26[0.08-0.84] ; p=0.02])$.

\section{Profile of cytokines in BALF by HLA-G expression}

We analyzed the correlation between dosage of cytokines in BALF and plasma sHLA-G level, and found a significant association only with TGF- $\beta$ level in BALF (Spearman correlation coefficient: $0.185, p=0.02$ ) (Table 3 ). In addition, lung HLA-G+ and HLA- patients did not differ in BALF cytokine profiles (Table 4).

\section{Discussion}

This prospective study showed a significant association of early repeated HLA-G expression in lung grafts from patients with stable LTX and functional stability on longterm follow-up. The role of graft HLA-G staining was analyzed in a multivariate model and was shown independent of other known factors associated with CLAD onset 


\section{Brugière et al}

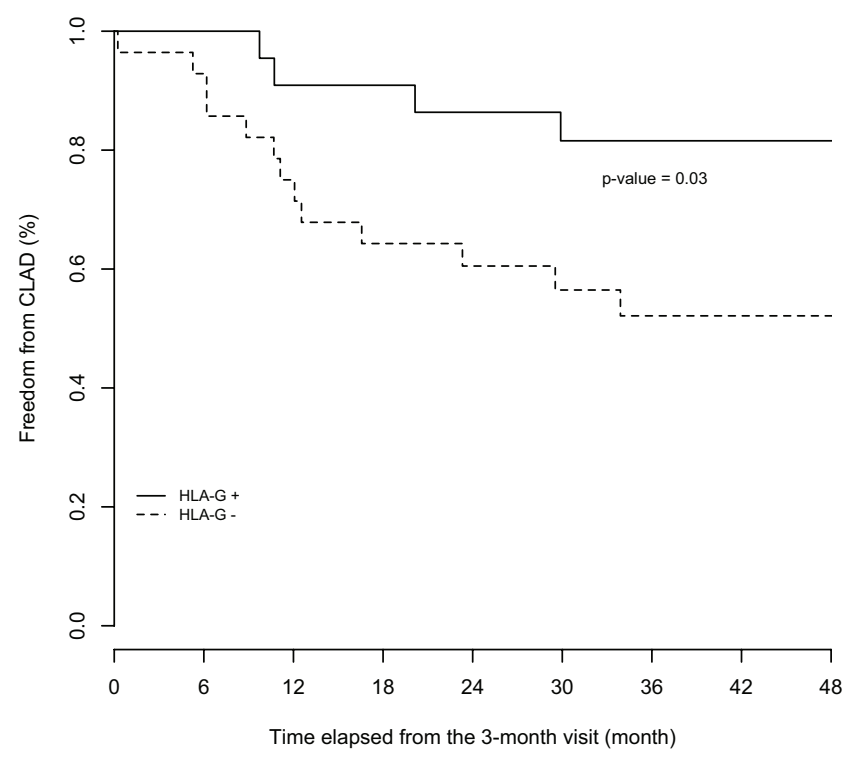

Figure 3: Kaplan-Meier curves comparing proportion of lung HLA-G + and HLA-G - patients free of chronic lung allograft dysfunction (CLAD), conditional on being alive and free of CLAD 3 months after transplantation. Freedom from CLAD was significantly higher for lung $\mathrm{HLA}-\mathrm{G}+$ patients compared to lung HLA-G - patients ( $p=0.03$, log-rank). HLA-G, human leukocyte antigen $\mathrm{G}$.

such as a high AR score or lymphocytic bronchiolitis. Furthermore, this graft tissue expression of HLA-G was inversely associated with post-LTx anti-HLA IgG Abs production, which suggests a protective role of HLA-G in humoral immunity.

In a previous cross-sectional study, bronchial HLA-G expression was more frequently expressed in LTx recipi-

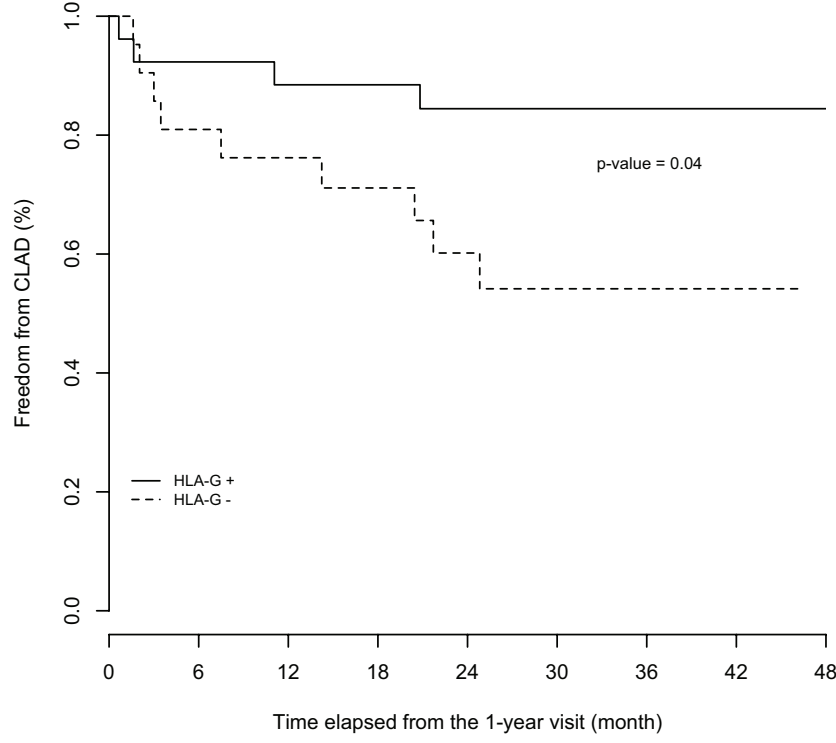

Figure 4: Kaplan-Meier curves comparing proportion of lung HLA-G + and HLA-G - patients free of chronic lung allograft dysfunction (CLAD), conditional on being alive and free of CLAD 12 months after transplantation. Freedom from CLAD was significantly higher for lung $\mathrm{HLA}-\mathrm{G}+$ patients compared to lung HLA-G - patients ( $p=0.04$, log-rank). HLA-G, human leukocyte antigen $\mathrm{G}$.

ents with stable condition at the date of biopsy (19). Our clinical study extends these findings, showing an association of graft HLA-G expression with a subsequent functional stability observed at a median time of more than 3 years. Our results are comparable to those found in longitudinal heart Tx studies, showing that graft HLA-G expression was predictive of a low risk of developing chronic rejection during follow-up $(7,13)$. Interestingly similar to our observed

Table 3: Univariate and multivariable Cox models

\begin{tabular}{lccc}
\hline Variable & HR & $95 \% \mathrm{Cl}$ & $\mathrm{p}-\mathrm{Value}$ \\
\hline Univariate analysis of factors associated with CLAD occurrence (Cox model) & & \\
Lymphocytic bronchiolitis & 2.45 & $0.88-6.78$ & 0.085 \\
Primary graft dysfunction & 1.10 & $0.73-1.66$ & 0.65 \\
Acute rejection score & 1.31 & $0.92-1.87$ & 0.13 \\
Anti-HLA Abs & 1.91 & $0.61-5.96$ & 0.26 \\
Procedure & 0.84 & $0.29-2.43$ & 0.75 \\
Graft HLA-G expression & 0.17 & $0.05-0.60$ & 0.006 \\
Multivariate analysis of factors associated with CLAD occurrence (Cox model) & & $0.67-8.43$ \\
Lymphocytic bronchiolitis & 2.38 & $0.72-1.65$ & 0.18 \\
Acute rejection score & 1.09 & $0.03-0.58$ & 0.68 \\
Graft HLA-G expression & 0.13 & 0.008 \\
\hline
\end{tabular}

95\% Cl, 95\% confidence interval; HR, hazard ratio.

PGD, primary graft dysfunction (PGD 3 vs. PGD 0-2) (1); AR score, acute rejection score, defined by the number of biopsy-proven cellular AR episodes during the first 12 months after lung transplantation (LTX) (25); Anti-HLA Abs, anti-HLA antibodies detected against HLA Class I and Class II antigen and identified by single-antigen flow-beads Luminex assay at visit 1 at 3 months (V1) or at visit 2 at 12 months (V2) (yes/no); Lymphocytic bronchiolitis (21) (yes/no); Procedure: surgical procedure (single LTx vs. bilateral LTx); Graft HLA-G expression: lung HLA-G+ (positive) versus lung HLA-G - (negative) patients; Lung HLA-G + (positive) patients if transbronchial biopsies at $\mathrm{V} 1$ or at $\mathrm{V} 2$ were considered HLA-G positive, with bronchial epithelium stained with MEM-G/2 mAb. The remaining patients were considered lung HLA-G negative. Statistical significance if $p<0.05$. 
HLA-G Is Associated With Lung Graft Acceptance

Table 4: Profiles of cytokines in BALF according to HLA-G expression in lung tissue or soluble HLA-G levels in plasma

\begin{tabular}{|c|c|c|c|c|c|}
\hline $\begin{array}{l}\text { Cytokine } \\
\text { (pg/mL) }\end{array}$ & $\begin{array}{l}\text { Lung HLA-G+ group } \\
\text { mean (SD) }\end{array}$ & $\begin{array}{c}\text { Lung HLA-G- group } \\
\text { mean (SD) }\end{array}$ & p-Value & $\begin{array}{l}\text { Correlation coefficient between } \\
\text { plasma sHLA-G and cytokines }\end{array}$ & $\mathrm{p}$-Value \\
\hline IL-6 & 6.77 (13.25) & $9.95(20.50)$ & 0.34 & 0.051 & 0.45 \\
\hline IL-8 & 538.51 (1834.33) & $277.53(448.53)$ & 0.32 & 0.068 & 0.47 \\
\hline MCP-1 & $252.83(649.80)$ & 214.61 (292.64) & 0.70 & 0.016 & 0.85 \\
\hline $\mid \mathrm{P}-10$ & $345.76(1188.40)$ & 450.58 (1586.07) & 0.70 & 0.038 & 0.69 \\
\hline RANTES & $1.50(4.69)$ & $3.08(10.72)$ & 0.32 & 0.128 & 0.15 \\
\hline IL-17 & $0.20(0.64)$ & $0.11(0.06)$ & 0.30 & -0.016 & 0.85 \\
\hline IL-10 & $1.53(3.85)$ & $1.84(4.92)$ & 0.72 & 0.062 & 0.99 \\
\hline GM-CSF & $0.50(1.24)$ & $0.25(1.04)$ & 0.27 & 0.069 & 0.08 \\
\hline TNF- $\alpha$ & $1.65(3.84)$ & $2.17(6.55)$ & 0.61 & 0.111 & 0.51 \\
\hline IFN- $\gamma$ & $0.41(1.95)$ & $0.08(0.12)$ & 0.22 & -0.001 & 0.99 \\
\hline CXCL9 & 124.94 (326.88) & 98.87 (269.39) & 0.65 & 0.069 & 0.47 \\
\hline IL-13 & $0.49(1.54)$ & $0.38(1.06)$ & 0.67 & -0.056 & 0.63 \\
\hline TGF- $\beta$ & $3.43(5.41)$ & $4.38(6.01)$ & 0.39 & 0.185 & 0.02 \\
\hline
\end{tabular}

Dosage of cytokines $(\mathrm{pg} / \mathrm{mL})$ are expressed as mean (SD).

Patients were classified as lung HLA-G positive (lung HLA-G+) patients if TBBx at visit 1 at 3 months (V1) or at visit 2 at 12 months (V2) were considered HLA-G positive, with bronchial epithelium stained with MEM-G/2 mAb. The remaining patients were considered lung HLA-G negative (lung $\mathrm{HLA}-\mathrm{G}-$ ).

The variation between all groups is calculated with one-way ANOVA and the Mann-Whitney test is used as post hoc test for significances of lung $\mathrm{HLA}-\mathrm{G}+$ versus lung $\mathrm{HLA}-\mathrm{G}$ - groups.

${ }^{1}$ Computed from a random-intercept model. Statistical significance if $p<0.05$

bronchiolar/bronchial cells expression, HLA-G staining was also detected in target cells of chronic rejection in other solid organ $T x$, such as biliary epithelial cells and tubular cells in liver and kidney $T x$, respectively $(8,9)$. Because graft rejection is largely mediated by immune effectors cells that are inhibited by HLA-G $(3,14-18)$, these findings could argue for a protective role of graft HLA-G expression against rejection. It could also be merely an association of HLA-G expression with a stable state. Why graft HLA-G expression is present in only certain stable LTx recipients remains unknown, and variable proportions of graft HLA-G expression have also been reported in liver (35\% HLA-G+), kidney (55\% HLA-G+) and heart (47\% HLA-G+) grafts in stable solid organ $T x$ recipients $(8,13)$. We observed a persistent graft expression of HLA-G in most lung HLA-G+ patients, as also observed in longitudinal heart Tx studies $(6,13)$. This finding may suggest that this expression can be activated during the first year after $T x$ and maintained by factors yet to be identified. Similarly, the HLA-G molecule can also be upregulated in other pathologic situations $(3,4,22-24,30-34)$. For example, in patients with neoplastic diseases, it has been demonstrated that HLA-G transcription and protein expression may be switched on in some tumor lesions and protect them against NK cytolysis (30-33), and is correlated with unfavorable outcome (4), which suggests a role of HLA-G neo-expression in tumoral escape from immune survey.

In contrast, some lung HLA-G+ patients $(n=5)$ developed CLAD from 9 to 25 months post-Tx, which corresponds to false-positive results of HLA-G as a predictor of graft acceptance. Among them, four displayed an intensity of HLA-G staining of ++ in one TBBx (Table 2 ), suggesting no relationship between the intensity of staining and associa- tion with CLAD occurrence. In the same way, rare graft HLA-G staining has been reported in heart Tx recipients with rejection (13), so the pathway of HLA-G expression may be, although infrequently, upregulated during rejection processes.

We found no association of bronchial HLA-G expression and the pathologic grading of pulmonary allograft rejection of ISHLT (21), which includes lymphocytic bronchiolitis lesions. This finding seems notable because HLA-G expression has been found in inflammatory diseases, such as inflammatory bowel disease or rhumatoic disease, but with a highly variable detection depending on type of inflammatory processes $(3,4,34)$. In LTX recipients, our results suggest that bronchial HLA-G expression can be upregulated independently of lymphocytic bronchiolitis-related inflammation.

In our study, plasma sHLA-G level did not predict graft acceptance during long-term follow-up. In contrast, we observed increased plasma sHLA-G levels at V2 in patients with subsequent CLAD during follow-up. These results disagree with previous studies of other solid organ Tx recipients, which reported high plasma sHLA-G values in heart- or liver-kidney $\mathrm{Tx}$ recipients with graft acceptance $(9,10,35)$. Furthermore, we did not observe any association of plasma sHLA-G levels and positive HLA-G staining of BECs in graft tissue, that is, between peripheral and graft expression of HLA-G. Several hypotheses for LTX are as follows: (i) The initial event of rejection is localized in the graft, and peripheral expression of HLA-G might be independent of in situ graft expression. This hypothesis agrees with recent results in asthma disease (36) showing no association of plasma sHLA-G levels and airway 


\section{Brugière et al}

pathology, but an association of airway HLA-G expression and asthma disease; (ii) The increase in plasma sHLA-G level during CLAD could result from HLA-G secretion by circulating immune cells of the recipient such as T cells (37) and monocytes (38), the main HLA-G producer in the peripheral blood, reflecting an attempt to stop the local process of rejection. This pathway needs to be confirmed, and additional analysis of graft HLA-G expression with dosage of sHLA-G in BALF could be of interest; (iii) Membrane-bound and sHLA-G molecules act differently on cytokine production (39), which results in a differential cytokinic environment that may account for the distinct association of both HLA-G forms with graft follow-up.

An interesting finding was the significantly lower proportion of patients with Luminex-detected anti-HLA Abs in those with graft HLA-G expression, which reflects a potential protective role of HLA-G against humoral immunity in LTX. This observation can be related to previous findings in heart Tx patients showing an association of sHLA-G expression and $\mathrm{C} 4 \mathrm{~d}$ staining associated with antibody-mediated rejection (40). It also agrees with the inhibitory role of HLA-G in proliferation, differentiation and antibody secretion of $B$ cells recently reported (41). Nevertheless, because HLA-G expression still had a protective effect on CLAD occurrence, whether the Cox model included anti-HLA Abs or not, our results also suggest that HLA-G expression seems at least in part independent of humoral immunity.

We examined the cytokine profile in BALF by both concomitant plasma sHLA-G levels and bronchial expression of $H L A-G$. Only increased TGF- $\beta$ level in BALF was associated with increased plasma sHLA-G level, whereas we found no alteration in levels of other cytokines by plasma sHLA-G level. This seems consistent with our finding of higher plasma sHLA-G levels in patients with CLAD at last-follow-up because TGF- $\beta$ is known to be profibrotic, and its presence in BALF has been associated with CLAD occurrence (42). Otherwise, the cytokine profile in BALF was not associated with bronchial expression of HLA-G in patients. HLA-G expression in the bronchial epithelium may not depend on inflammatory cytokines, such as Th2-associated cytokines, as was recently reported in a model of primary culture of BECs (43). Hence, increased HLA-G expression in the graft may be the initial event in some stable LTx recipients, with a role in dampening the inflammatory response.

Our findings have several limitations. First, HLA-G staining in the lung graft was not analyzed in the case of communityacquired viral infections, which are well known to induce HLA-G expression (19,22-24), as was previously shown in bronchial epithelium (19). Because viral infection is a frequent cause of decreased lung function in the LTx population (44), this issue highly limits the clinical usefulness of graft HLA-G expression in real-life practice during follow-up of LTx recipients. Also, analysis of HLA-G staining needs to exclude some TBBx with inadequate bronchiolar epithelium samples, such as those classified as Bx by the ISHLT grading (21), and this possible sampling bias should be recognized as a limitation in our study.

In conclusion, detection of graft HLA-G expression appears to indicate a low risk of developing subsequent CLAD in this prospective cohort of LTx recipients. Nevertheless, caution should be paid in interpreting this potential marker because we observed false-positive results and because strict exclusion of ongoing viral infection seems a sine qua non condition for interpreting HLA-G bronchial expression. Conversely, the plasma sHLA-G level, as a potential noninvasive marker of graft acceptance in other solid organ Tx, was not associated with graft outcome in our cohort. Future investigations should elucidate the precise mechanisms that might contribute to HLA-G expression after LTX.

\section{Acknowledgments}

This study was supported by AP-HP (Assistance Publique des Hôpitaux de Paris), CEA, and Vaincre la Mucoviscidose.

\section{Disclosure}

The authors of this manuscript have no conflicts of interest to disclose as described by the American Journal of Transplantation.

\section{References}

1. Kotloff RM, Thabut G. Lung transplantation. Am J Respir Crit Care Med 2011; 184: 159-171.

2. Girlanda R, Kirk AD. Frontiers in nephrology: Immune tolerance to allografts in humans. J Am Soc Nephrol 2007; 18: 2242-2251.

3. Loustau M, Wiendl H, Ferrone S, Carosella ED. HLA-G 2012 conference: The 15-year milestone update. Tissue Antigens 2013; 81: 127-136.

4. Carosella ED, Favier B, Rouas-Freiss N, Moreau P, LeMaoult J. Beyond the increasing complexity of the immunomodulatory HLAG molecule. Blood 2008; 111: 4862-4870.

5. Hviid TVF. HLA-G in human reproduction: Aspects of genetics, function and pregnancy complications. Hum Reprod Update 2006; 12: 209-232.

6. Lila N, Carpentier A, Amrein C, Khalil-Daher I, Dausset J, Carosella ED. Implication of HLA-G molecule in heart-graft acceptance. Lancet 2000; 355: 2138

7. Lila N, Amrein C, Guillemain R, et al. Human leukocyte antigen-g expression after heart transplantation is associated with a reduced incidence of rejection. Circulation 2002; 105: 1949-1954.

8. Creput $C$, Durrbach A, Menier $C$, et al. Human leukocyte antigen-g (HLA-G) expression in biliary epithelial cells is associated with allograft acceptance in liver-kidney transplantation. J Hepatol 2003; 39: 587-594.

9. Creput C, Le Friec G, Bahri R, et al. Detection of HLA-G in serum and graft biopsy associated with fewer acute rejections following combined liver-kidney transplantation: Possible implications for monitoring patients. Hum Immunol 2003; 64: 1033-1038. 
HLA-G Is Associated With Lung Graft Acceptance

10. Luque J, Torres MI, Aumente MD, et al. Soluble HLA-G in heart transplantation: Their relationship to rejection episodes and immunosuppressive therapy. Hum Immunol 2006; 67: 257-263.

11. Qiu J, Terasaki PI, Miller J, Mizutani K, Cai J, Carosella ED. Soluble HLA-G expression and renal graft acceptance. Am J Transplant 2006; 6: 2152-2156.

12. Crispim JC, Duarte RA, Soares CP, et al. Human leukocyte antigen$\mathrm{g}$ expression after kidney transplantation is associated with a reduced incidence of rejection. Transpl Immunol 2008; 18: 361367.

13. Sheshgiri R, Rouas-Freiss N, Rao V, et al. Myocardial HLA-G reliably indicates a low risk of acute cellular rejection in heart transplant recipients. J Heart Lung Transplant 2008; 27: 522-527.

14. Le Rond S, Azema C, Krawice-Radanne I, et al. Evidence to support the role of HLA-G5 in allograft acceptance through induction of immunosuppressive/regulatory T cells. J Immunol 2006; 176: 3266-3276.

15. Naji A, Le Rond S, Durrbach A, et al. CD3+ CD4low and CD3+ CD8low are induced by HLA-G: Novel human peripheral blood suppressor T-cell subsets involved in transplant acceptance. Blood 2007; 110: 3936-3948.

16. Riteau B, Rouas-Freiss N, Menier C, Paul P, Dausset J, Carosella ED. HLA-G2, -G3, and -G4 isoforms expressed as nonmature cell surface glycoproteins inhibit NK and antigen-specific CTL cytolysis. $\mathrm{J}$ Immunol 2001; 166: 5018-5026.

17. Lila N, Rouas-Freiss N, Dausset J, Carpentier A, Carosella ED. Soluble HLA-G protein secreted by allo-specific CD4+ T cells suppresses the allo-proliferative response: $\mathrm{A} C D 4+\mathrm{T}$ cell regulatory mechanism. Proc Natl Acad Sci U S A 2001; 98: 12150-12155.

18. Contini $P$, Ghio $M$, Poggi $A$, et al. Soluble HLA-A, $-B,-C$ and $-G$ molecules induce apoptosis in T and NK CD8+ cells and inhibit cytotoxic T cell activity through CD8 ligation. Eur J Immunol 2003; 33: $125-134$.

19. Brugiere O, Thabut G, Pretolani M, et al. Immunohistochemical study of HLA-G expression in lung transplant recipients. Am J Transplant 2009; 9: 1427-1438.

20. Brugiere O, Thabut G, Mal H, et al. Exhaled NO may predict the decline in lung function in bronchiolitis obliterans syndrome. Eur Respir J 2005; 25: 813-819.

21. Stewart S, Fishbein MC, Snell Gl, et al. Revision of the 1996 working formulation for the standardization of nomenclature in the diagnosis of lung rejection. J Heart Lung Transplant 2007; 26: 1229-1242.

22. Onno M, Pangault $C$, Le Friec G, Guilloux V, Andre P, Fauchet $R$. Modulation of HLA-G antigens expression by human cytomegalovirus: Specific induction in activated macrophages harboring human cytomegalovirus infection. J Immunol 2000; 164: 64266434.

23. Lafon M, Prehaud C, Megret F, et al. Modulation of HLA-G expression in human neural cells after neurotropic viral infections. J Virol 2005; 79: 15226-15237.

24. LeBouder F, Khoufache K, Menier C, et al. Immunosuppressive HLA-G molecule is upregulated in alveolar epithelial cells after influenza A virus infection. Hum Immunol 2009; 70: 1016-1019.

25. Swarup R, Allenspach LL, Nemeh HW, Stagner LD, Betensley AD. Timing of basiliximab induction and development of acute rejection in lung transplant patients. J Heart Lung Transplant 2011; 30: 1228-1235.

26. Cooper JD, Billingham M, Egan $T$, et al. A working formulation for the standardization of nomenclature and for clinical staging of chronic dysfunction in lung allografts. J Heart Lung Transplant 1993; 12: 713-721.
27. Verleden GM, Vos R, Verleden SE, et al. Survival determinants in lung transplant patients with chronic allograft dysfunction. Transplantation 2011; 92: 703-708.

28. Rizzo R, Farina I, Bortolotti D, et al. HLA-G may predict the disease course in patients with early rheumatoid arthritis. Hum Immunol 2013; 74: 425-432.

29. Kennedy VE, Todd JL, Palmer SM. Bronchoalveolar lavage as a tool to predict, diagnose and understand bronchiolitis obliterans syndrome. Am J Transplant 2013; 13: 552-561.

30. Bukur J, Rebmann V, Grosse-Wilde $\mathrm{H}$, et al. Functional role of human leukocyte antigen-G up-regulation in renal cell carcinoma. Cancer Res 2003; 63: 4107-4111.

31. Wiendl $H$, Mitsdoerffer $M$, Hofmeister $V$, et al. A functional role of HLA-G expression in human gliomas: An alternative strategy of immune escape. J Immunol 2002; 168: 4772-4780.

32. Paul $P$, Rouas-Freiss N, Khalil-Daher I, et al. HLA-G expression in melanoma: A way for tumor cells to escape from immunosurveillance. Proc Natl Acad Sci U S A 1998; 95: 4510-4515.

33. Rouas-Freiss N, Moreau P, Ferrone S, Carosella ED. HLA-G proteins in cancer: Do they provide tumor cells with an escape mechanism? Cancer Res 2005; 65: 10139-10144.

34. Torres MI, Le Discorde M, Lorite $P$, et al. Expression of HLA-G in inflammatory bowel disease provides a potential way to distinguish between ulcerative colitis and Crohn's disease. Int Immunol 2004; 16: 579-583.

35. Lila N, Amrein C, Guillemain R, Chevalier P, Fabiani JN, Carpentier A. Soluble human leukocyte antigen-G: A new strategy for monitoring acute and chronic rejection after lung transplantation. J Heart Lung Transplant 2007; 26: 421-422.

36. Nicodemus-Johnson J, Laxman B, Stern RK, et al. Maternal asthma and microRNA regulation of soluble HLA-G in the airway. $J$ Allergy Clin Immunol 2013; 131: 1496-1503.

37. Le Rond S, Le Maoult J, Creput $\mathrm{C}$, et al. Alloreactive $\mathrm{CD}^{+}$and $\mathrm{CD}^{+} \mathrm{T}$ cells express the immunotolerant HLA-G molecule in mixed lymphocyte reactions: In vivo implications in transplanted patients. Eur J Immunol 2004; 34: 649-660.

38. Moreau P, Adrian-Cabestre F, Menier C, et al. IL-10 selectively induces HLA-G expression in human trophoblasts and monocytes. Int Immunol 1999; 11: 803-811.

39. Kanai T, Fujii T, Kozuma $S$, et al. Soluble HLA-G influences the release of cytokines from allogeneic peripheral blood mononuclear cells in culture. Mol Hum Reprod 2001; 7: 195-200.

40. Sheshgiri R, Rao V, Mociornita A, Ross HJ, Delgado DH. Association between HLA-G expression and C4d staining in cardiac transplantation. Transplantation 2010; 89: 480-481.

41. Naji A, Menier C, Morandi F, et al. Binding of HLA-G to ITIM-bearing Ig-like receptors suppresses B cell responses. J. Immunol 2014; 15: 1536-1546.

42. Elssner A, Jaumann F, Dobmann S, et al. Munich Lung Transplant Group. Elevated levels of interleukine-8 and transforming growth factor-beta in bronchoalveolar lavage fluid from patients with bronchiolitis obliterans sybdrome: Proinflammatory role of bronchial epithelial cells. Transplantation 2000; 70: 362367.

43. White SR, Loisel DA, Stern R, Laxman B, Floreth T, Marroquin BA. Human leukocyte antigen- $G$ expression in differentiated human airway epithelial cells: Lack of modulation by Th2-associated cytokines. Respir Res 2013; 14: 4.

44. Kumar D, Husain S, Hong Chen M, et al. Prospective molecular surveillance study evaluating the clinical impact of communityacquired respiratory viruses in lung transplant recipients. Transplantation 2010; 89: 1028-1033. 